\title{
ESPECIAÇÃO E QUIMISSORÇÃO DE Pb(II) EM REJEITO DE CAULIM
}

\author{
Marta Helena Tavares Pinheiro*, Vanda Porpino Lemos e Kelly das G. Fernandes Dantas \\ Faculdade de Química, Instituto de Ciências Exatas e Naturais, Universidade Federal do Pará, Cidade Universitária Prof. José \\ Silveira Netto, Setor Básico, Rua Augusto Corrêa, 1, 66075-110 Belém - PA, Brasil \\ Taynara Lima Valentim \\ Faculdade de Engenharia Química, Instituto Tecnológico, Universidade Federal do Pará, Cidade Universitária Prof. José Silveira \\ Netto, Setor Profissional, Rua Augusto Corrêa, 1, 66075-110 Belém - PA, Brasil
}

Recebido em 18/6/12; aceito em 28/8/12; publicado na web em 24/1/13

\begin{abstract}
SPECIATION AND CHEMISORPTION OF Pb(II) ON KAOLIN WASTE. This study assesses the adsorption of $\mathrm{Pb}$ (II) on natural kaolin waste $\left(\mathrm{KR}_{\text {natural }}\right)$ and on that treated with $3 \mathrm{~mol} \mathrm{~L}^{-1} \mathrm{H}_{2} \mathrm{SO}_{4}$ and $\mathrm{HCl}$. Equilibrium and thermodynamic parameters were determined. The results indicate that the values of $\mathrm{CEC}$, specific area and $\mathrm{SiO}_{2} / \mathrm{Al}_{2} \mathrm{O}_{3}$ ratio $\left(4.6-6.0 \mathrm{cmol} \mathrm{kg}^{-1}, 14.0-16.0 \mathrm{~m}^{2} \mathrm{~g}^{-1}\right.$ and 1.16-1.30, respectively) vary only slightly for the adsorbents; the concentration of $\mathrm{Pb}^{2+}$ is much higher than that of other species $\left(\mathrm{PbOH}^{+}\right.$and $\left.\mathrm{Pb}(\mathrm{OH})_{2}\right)$. The values of $R_{\mathrm{L}}, \Delta G^{\mathrm{o}}, \Delta H^{\mathrm{o}}$ and $\Delta S^{\mathrm{o}}$ are typical of feasible, spontaneous, exothermic and ordered adsorption. The chemisorption on $\mathrm{KR}_{\text {natural }}$ is more feasible and ordered.
\end{abstract}

Keywords: lead; kaolinite; adsorption.

\section{INTRODUÇÃO}

A migração de elementos tóxicos nos minerais presentes em solos e sedimentos é bastante influenciada por processos de adsorção na interface sólido-solução. ${ }^{1,2}$ Os minerais de argila desempenham papel importante nestes processos devido apresentarem propriedades adequadas à retenção de espécies químicas nos sítios de cargas negativas permanentes e nos sítios de cargas variáveis de acordo com o $\mathrm{pH} .{ }^{3}$ Este aspecto é muito bem reconhecido, conforme demonstrado em vários estudos experimentais ${ }^{4-6}$ e modelos teóricos a partir de interações de grupos superficiais de minerais de argilas com cátions metálicos. ${ }^{7-9}$

A contaminação de metais pesados no meio ambiente é um problema de grande preocupação global devido especialmente à bioacumulação nos organismos. A absorção de materiais contaminados com chumbo no corpo humano se inicia principalmente por via oral, seguindo para o estômago, onde as espécies que contêm chumbo são liberadas e depois são absorvidas no intestino e distribuídas através do sangue para vários órgãos. As crianças são mais vulneráveis aos efeitos tóxicos do chumbo do que os adultos. Isto é devido ao comportamento das crianças, que muitas vezes colocam suas mãos sujas e brinquedos na boca ou nariz, aumentando a possibilidade de ingestão de partículas de chumbo. ${ }^{10-13}$

Vários métodos são utilizados para remoção de cátions $\mathrm{M}^{\mathrm{n}+}$ de metais pesados a partir de soluções aquosas, incluindo reações de precipitação química, troca iônica, extração com solvente, ultrafiltração, osmose reversa e adsorção por minerais de argila, por exemplo, caulinita e seus derivados. ${ }^{14-16}$

A caulinita é o mineral predominante no caulim, que é uma rocha sedimentar encontrada em vários depósitos do mundo. No Brasil, as reservas de caulim se concentram nos estados do Pará, Amapá e Amazonas, sendo o minério já beneficiado disponibilizado para os mercados interno e externo. ${ }^{17}$ No processo de beneficiamento do caulim por empresas paraenses é gerado elevado volume de rejeito, que é depositado em extensas e onerosas lagoas de sedimentação. Quando as lagoas são rompidas acidentalmente, ocorre invasão de grande volume de rejeito em direção aos rios da região, provocando morte de peixes.

*e-mail: mhtp@ufpa.br
Estes problemas têm incentivado pesquisadores de vários locais em utilizar os rejeitos de caulim do estado do Pará como matéria-prima na síntese de zeólitas e em outros tipos de aplicação, como na produção de refratários e cerâmicas avançadas, metacaulim (pozolana) em cimento Portland, mullita para fabricação de chamota e refratários. ${ }^{18-20}$

Neste estudo foi avaliada a adsorção do $\mathrm{Pb}$ (II) em caulinita a partir de rejeito de caulim do estado do Pará, que foi selecionado como material de partida devido apresentar elevada pureza e contribuir no seu reaproveitamento e na minimização de problemas ao meio ambiente. Nos experimentos de adsorção, o rejeito de caulim foi utilizado em sua forma natural e tratado com soluções de $\mathrm{H}_{2} \mathrm{SO}_{4}$ e $\mathrm{HCl} 3 \mathrm{~mol}$ $\mathrm{L}^{-1}$ a fim de se modificarem as propriedades estrutural e textural da caulinita e empregarem os materiais produzidos nas avaliações sobre especiação do $\mathrm{Pb}(\mathrm{II})$ nas interfaces caulinitas-soluções; viabilidade e espontaneidade dos processos de adsorção, com base no fator de separação e variação de energia livre de Gibbs; calor das reações e grau de desordem dos processos de adsorção a partir de cálculos das variações de entalpia e entropia, respectivamente.

\section{PARTE EXPERIMENTAL}

\section{Preparação dos adsorventes}

O material de partida usado foi o rejeito de caulim produzido pelo processamento de uma indústria de beneficiamento de caulim, localizada entre as cidades de Ipixuna e Rio Maria, no estado do Pará. Amostras de $5 \mathrm{~g}$ do rejeito de caulim in natura $\left(\mathrm{KR}_{\text {natural }}\right)$ foram ativadas com soluções de ácido sulfúrico $\left(\mathrm{H}_{2} \mathrm{SO}_{4}\right)$ e ácido clorídrico $(\mathrm{HCl})$ em concentrações iguais a $3 \mathrm{~mol} \mathrm{~L}^{-1}$ (denominadas como $\mathrm{KR}_{\mathrm{H} 2 \mathrm{SO}-3}$ e $\mathrm{KR}_{\mathrm{HCl}-3}$ ) por $24 \mathrm{~h} \mathrm{em} \mathrm{T}=80^{\circ} \mathrm{C}$. Posteriormente foram filtradas a vácuo, lavadas com água deionizada até teste negativo para sulfato e cloreto e colocadas para secar em estufa a $50{ }^{\circ} \mathrm{C} \pm 5$ por $10 \mathrm{~h}$.

\section{Caracterização dos adsorventes}

Em estudo anterior foi indicado que os materiais $\mathrm{KR}_{\text {natural }}$, $\mathrm{KR}_{\mathrm{H} 2 \mathrm{SO}-3-3}$ e $\mathrm{KR}_{\mathrm{HCl}-3}$ são constituídos predominantemente por caulinita, 
que as suas cargas estruturais são negativas (cargas dependentes do $\mathrm{pH}$ estimadas a partir de $\left.\Delta \mathrm{pH}=\mathrm{pH}_{\mathrm{KCl}}-\mathrm{pH}_{\mathrm{H} 2 \mathrm{O}}\right)^{21}$

Neste estudo as amostras foram caracterizadas por difração de raios $\mathrm{X}$, microscopia eletrônica de varredura combinada com espectroscopia de dispersão de energia de raios $\mathrm{X}$ (MEV/EDX), capacidade de troca catiônica (CTC) e área específica (S).

As análises por DRX (método do pó) foram realizadas em um difratômetro X' Pert PRO MPD (PW 3040/60) PANalytical, usando filtro de $\mathrm{Ni}$, radiação $\mathrm{Cu} \mathrm{K} \alpha$, angulo $2 \theta$ no intervalo de 5 a $75^{\circ}$, com varredura de $0,02^{\circ} \mathrm{s}^{-1}$, operando em $40 \mathrm{kV}$ e $40 \mathrm{~mA}$. As fases cristalinas foram identificadas usando o software X'PertHighScore versão $2.1 \mathrm{~b}$.

As análises por MEV/EDX foram realizadas em microscópio eletrônico de varredura, modelo LEO-1430. As amostras utilizadas nas análises foram previamente metalizadas com uma película de ouro de espessura média de $15 \mathrm{~nm}$ e os resultados das análises foram dados a partir de imagens geradas por elétrons secundários com voltagens de $20 \mathrm{kV}$ e registrados em alta resolução.

A capacidade de troca catiônica (CTC) foi determinada pelo método acetato de sódio seguindo as etapas: pesagem de $1 \mathrm{~g}$ de cada amostra, adição em excesso de solução de acetato de sódio 1 mol L-1, agitação por 5 min, centrifugação, lavagens do resíduo com álcool isopropílico, adição de acetato de amônio $1 \mathrm{~mol} \mathrm{~L}^{-1}$, visando a substituição do $\mathrm{Na}^{+}$adsorvido pelo $\mathrm{NH}_{4}^{+}$, análise do $\mathrm{Na}^{+}$liberado por FAAS. $^{21}$

A área específica $(\mathrm{S})$ foi determinada usando-se o nitrogênio como sorbato em temperatura de $77 \mathrm{~K}$ e as medidas efetuadas em um instrumento Quantachrome/Nova-1200/5.01. O cálculo da S foi efetuado pelo método Brunauer-Emmett-Teller (BET). ${ }^{22}$

\section{Experimentos de adsorção}

Os experimentos para estimar a capacidade de adsorção do rejeito de caulim natural e tratado com soluções ácidas foram efetuados em batelada. Solução estoque $1000 \mathrm{mg} \mathrm{L}^{-1}$ de íon chumbo foi preparada a partir do sal $\mathrm{Pb}\left(\mathrm{NO}_{3}\right)_{2}$ de grau analítico Merck (São Paulo, Brasil) em água deionizada. Foram preparadas suspensões aquosas contendo 0,5 $\mathrm{g}$ dos adsorventes e 50,0 mL de solução de $\mathrm{Pb}$ (II) em concentrações que variaram entre 40 a $100 \mathrm{mg} \mathrm{L}^{-1}$. Os experimentos foram realizados a 30,40, 45 e $50{ }^{\circ} \mathrm{C}$ e sob agitação empregando-se um agitador horizontal, Lab Line orbit shaker trabalhando na frequência de $150 \mathrm{rpm}$ por $16 \mathrm{~h}$. Após agitação, as suspensões foram centrifugadas a $500 \mathrm{rpm}$ por $30 \mathrm{~min}$. As concentrações de equilíbrio (Ce) foram medidas por espectrofotometria de absorção atômica com atomização com chama (FAAS), utilizando-se um espectrômetro Varian Spectra AA 200 e lâmpada de cátodo oco de chumbo $(\lambda=217,00 \mathrm{~nm})$ no sobrenadante de cada ponto. A capacidade de adsorção dos adsorventes qe (mg do íon $\mathrm{Pb}(\mathrm{II}) / \mathrm{g}$ de adsorvente) foi calculada empregando-se a Equação 1:23

$$
\mathrm{qe}\left(\mathrm{mg} \mathrm{g}^{-1}\right)=\frac{(\mathrm{Ci}-\mathrm{Ce})}{\mathrm{m}(\mathrm{g})} \times \mathrm{V}
$$

onde, Ci e Ce são as concentrações inicial e de equilíbrio $\left(\mathrm{mol} \mathrm{L}^{-1}\right)$, respectivamente; $\mathrm{V}$ é o volume da solução (L), m é a massa do adsorvente $(\mathrm{g})$.

\section{Isotermas de adsorção}

A dinâmica dos processos de adsorção de espécies químicas em solução em materiais sólidos, como minerais de argila, depende da distribuição destas espécies entre as fases sólidas e líquidas. O equilíbrio de adsorção é avaliado a partir das isotermas de adsorção, que são representadas pelas concentrações das espécies químicas nas fases sólidas em relação às das fases líquidas. ${ }^{24}$

Os dados experimentais de adsorção foram ajustados aos modelos de isotermas de adsorção de Langmuir e Freundlich, usando as equações não lineares. A isoterma de Langmuir propõe um mecanismo de adsorção em superfícies homogêneas, assumindo a uniformidade da superfície dos adsorventes e todos os sítios ativos de adsorção são energeticamente idênticos. O modelo de Langmuir é descrito pela Equação 2:25

$$
\mathrm{q}_{\mathrm{e}}=\frac{\mathrm{q}_{\text {máx }} \mathrm{K}_{\mathrm{L}} \mathrm{C}_{\mathrm{e}}}{1+\mathrm{K}_{\mathrm{L}} \mathrm{C}_{\mathrm{e}}}
$$

onde $\mathrm{q}_{\mathrm{e}}$ é quantidade de soluto adsorvido por unidade de massa de adsorvente $\left(\mathrm{mg} \mathrm{g}^{-1}\right), \mathrm{C}_{\mathrm{e}}$ é concentração no equilíbrio do soluto em solução $\left(\mathrm{mol} \mathrm{L}^{-1}\right), q_{\text {maxx }}$ é o parâmetro que representa capacidade de adsorção na monocamada ( $\mathrm{mg} \mathrm{g}^{-1}$ ) e $K_{L}$ é a constante de Langmuir, parâmetro relacionado com a energia de adsorção e dependente da temperatura $\left(\mathrm{L} \mathrm{mol}^{-1}\right)$.

O grau de desenvolvimento e da espontaneidade da reação de adsorção podem ser obtidos a partir da avaliação do parâmetro de equilíbrio ou fator de separação $\mathrm{R}_{\mathrm{L}}$ (Equação 3), que indica se a reação de adsorção é favorável ou desfavorável, por meio da relação: ${ }^{26}$

$$
\mathrm{R}_{\mathrm{L}}=\frac{1}{1+\mathrm{K}_{\mathrm{L}} \mathrm{C}_{\mathrm{i}}}
$$

Na Equação 3, $\mathrm{C}_{\mathrm{i}}\left(\mathrm{mol} \mathrm{L}^{-1}\right)$ é a concentração inicial do íon e $\mathrm{K}_{\mathrm{L}}$ é a constante de afinidade estimada pela equação de Langmuir. $\mathrm{O}$ valor de $R_{L}$ entre 0 e 1 indica adsorção favorável.

A adsorção é considerada favorável se $\left(0<\mathrm{R}_{\mathrm{L}}<1\right)$, desfavorável se $\left(R_{L}>1\right)$, linear $\left(R_{L}=1\right)$ e irreversível $\left(R_{L}=0\right)$.

A isoterma de Freundlich é aplicada para descrever o equilíbrio de sistemas heterogêneos. A forma não linearizada é expressa pela Equação 4:27

$$
q e=K_{F} C_{e}^{1 / n}
$$

onde qe representa a quantidade de soluto na fase sólida no equilíbrio, $\mathrm{C}_{\mathrm{e}}$ é a concentração da fase líquida no equilíbrio, $\mathrm{n}$ indica, quantitativamente, a reatividade dos sítios energéticos do material e $\mathrm{K}_{\mathrm{F}}(\mathrm{L}$ $\mathrm{g}^{-1}$ ) pode sugerir a adsorção do íon no material.

\section{Erros de adsorção}

Os dados de adsorção foram avaliados com base nos valores de erros calculados pela Equação 5:24,28-30

$$
\operatorname{Erro}(\%)=100 \times \sqrt{\sum_{i}^{p}\left(\frac{q_{\text {imodelo }}-q_{\text {i experimental }}}{q_{\text {i experimental }}}\right)^{2} \times\left(\frac{1}{p-1}\right)}
$$

onde, pé o número de suspensões utilizadas no processo de adsorção, $\mathrm{q}_{\mathrm{i} \text { modelo }}$ é a capacidade de adsorção calculada pelo modelo de isoterma usado e $\mathrm{q}_{\mathrm{i} \text { experimental }}$ a capacidade de adsorção experimental.

\section{Parâmetros termodinâmicos da reação de adsorção}

\section{Constante de equilíbrio}

A constante de equilíbrio de uma reação de adsorção pode ser calculada usando a constante de distribuição $\left(\mathrm{K}_{\mathrm{d}}\right)$ como descrita pela Equação 6:31-33

$$
\mathrm{K}_{\mathrm{d}}=\mathrm{qe} / \mathrm{Ce}
$$




\section{Variação de energia livre}

A relação geral entre a variação de energia livre padrão, $\Delta \mathrm{G}^{\mathrm{o}}$, e a variação de energia livre sob quaisquer outras condições, $\Delta \mathrm{G}$, é dada pela expressão:

$$
\Delta \mathrm{G}=\Delta \mathrm{G}^{\mathrm{o}}+\mathrm{RT} \ln \mathrm{K}
$$

onde, $\Delta \mathrm{G}$ é a variação de energia livre de Gibbs $\left(\mathrm{kJ}^{\mathrm{mol}}{ }^{-1}\right), \mathrm{R}$ é a constante universal dos gases $\left(8,314 \mathrm{~J} \mathrm{~mol}^{-1} \mathrm{~K}^{-1}\right)$, T é a temperatura absoluta $(\mathrm{K})$ e K é a constante aparente da reação de adsorção. No equilíbrio a variação de energia livre de um processo de adsorção é calculada pela Equação 8:

$$
\Delta \mathrm{G}^{\mathrm{o}}=-\mathrm{RT} \ln \mathrm{K}
$$

onde $\mathrm{K}$ é a constante $\mathrm{K}_{\mathrm{L}}$ de Langmuir ou a constante de distribuição, conforme foi indicado em alguns estudos sobre adsorção do chumbo em caulinitas. ${ }^{14,31-33} \mathrm{O}$ sinal de $\Delta \mathrm{G}^{\mathrm{o}}$ indica se a reação é espontânea ou não, em um sistema fechado em determinada temperatura.

\section{Entalpia e entropia}

$\mathrm{O}$ calor de adsorção $\left(\Delta \mathrm{H}^{\circ}\right)$ pode ser medido diretamente por titulação calorimétrica ou calculado a partir da relação linear de van't Hoff que envolve dados experimentais de adsorção em diferentes temperaturas, como foi determinado neste estudo. Esta relação é muito usada no cálculo dos valores de $\Delta \mathrm{H}^{\circ}$ e $\Delta \mathrm{S}^{\circ}$ e é expressa pela Equação 9:33-35

$$
\ln \mathrm{K}_{\mathrm{L}}=-\frac{\Delta \mathrm{H}^{\mathrm{o}}}{\mathrm{R}} \cdot \frac{1}{\mathrm{~T}}+\frac{\Delta \mathrm{S}^{\mathrm{o}}}{\mathrm{R}}
$$

Nessa equação, $\mathrm{R}$ é a constante universal dos gases, igual a $8,314 \mathrm{~J} \mathrm{~mol}^{-1} \mathrm{~K}^{-1}, \mathrm{~K}_{\mathrm{L}}\left(\mathrm{L} \mathrm{mol}^{-1}\right)$ é a constante de Langmuir a partir da equação linearizada.

\section{RESULTADOS E DISCUSSÃO}

\section{Caracterização dos adsorventes}

Os dados de DRX indicaram que a caulinita $\left(\mathrm{Al}_{2} \mathrm{O}_{3} 2 \cdot \mathrm{SiO}_{2} 2 \cdot \mathrm{H}_{2} \mathrm{O}\right)$ é o mineral presente nos adsorventes, (Figura $1 \mathrm{~S}$, material suplementar) e não mostram decréscimos aparentes nas intensidades dos picos nos valores de $2 \theta$ entre $10^{\circ} \mathrm{a} 70^{\circ}$, correspondentes às amostras de rejeito de caulim natural e tratadas com soluções de $\mathrm{H}_{2} \mathrm{SO}_{4}$ e $\mathrm{HCl} 3 \mathrm{~mol} \mathrm{~L}^{-1}$. Os decréscimos nas intensidades dos picos de DRX poderiam indicar o ataque das folhas octaédricas provocadas pela lixiviação com soluções ácidas. Esta característica é referida como delaminação. ${ }^{36}$

A delaminação da caulinita com tratamentos ácidos foi verificada pelos dados de MEV/EDX. O tratamento ácido provocou alteração dos aspectos morfológicos (Figura 2S, material suplementar), que é indicada pela esfoliação dos cristais empilhados da caulinita e aumento das razões $\mathrm{SiO}_{2} / \mathrm{Al}_{2} \mathrm{O}_{3}$ em relação à amostra sem tratamento ácido (Tabela 1). As razões $\mathrm{SiO}_{2} / \mathrm{Al}_{2} \mathrm{O}_{3}$ foram obtidas a partir da média

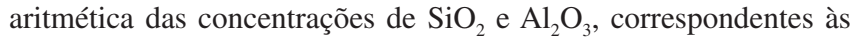
análises pontuais por EDX realizadas nas amostras. ${ }^{21}$

Os valores de CTC e $\mathrm{S}_{\mathrm{BET}}$ obtidos para amostra de rejeito de caulim (Tabela 1) encontram-se nos intervalos indicados para caulinita. ${ }^{32,37} \mathrm{Na}$ amostra $\mathrm{KR}_{\text {natural }}$ o valor de CTC é próximo do limite inferior desse intervalo, que é de 5 a $15 \mathrm{cmol}_{\mathrm{c}} \mathrm{kg}^{-1} \cdot{ }^{14,37} \mathrm{O}$ baixo valor de CTC obtido para $\mathrm{KR}_{\text {natural }}$ é compatível com o grau de cristalinidade e pureza do rejeito de caulim. Estudos anteriores sobre índices de cristalinidade e pureza de caulinitas brasileiras indicaram que a caulinita do Rio Capim tem índice de Hinckley $\mathrm{HI}=1,17$ e que este valor
Tabela 1. Propriedades químicas e $S_{\mathrm{BET}}$ das amostras de rejeito de caulim natural $\left(\mathrm{KR}_{\text {natural }}\right)$ e tratadas com soluções de $\mathrm{H}_{2} \mathrm{SO}_{4}$ e $\mathrm{HCl} 3 \mathrm{~mol} \mathrm{~L}^{-1}\left(\mathrm{KR}_{\mathrm{H} 2 \mathrm{SO}-3}\right.$ e $\mathrm{KR}_{\mathrm{HCl}-3}$ )

\begin{tabular}{ccccc}
\hline Amostras & $\mathrm{SiO}_{2} / \mathrm{Al}_{2} \mathrm{O}_{3}$ & $\begin{array}{c}\mathrm{CTC} \\
\left(\mathrm{cmol}_{\mathrm{c}} \mathrm{kg}^{-1}\right)\end{array}$ & $\mathrm{Al}_{2} \mathrm{O}_{3} / \mathrm{CTC}$ & $\begin{array}{c}\mathrm{S}_{\mathrm{BET}} \\
\left(\mathrm{m}^{2} \mathrm{~g}^{-1}\right)\end{array}$ \\
\hline $\mathrm{KR}_{\text {natural }}$ & 1,16 & 6,60 & 0,589 & 14,00 \\
$\mathrm{KR}_{\mathrm{H} 2 \mathrm{SO} 4-3}$ & 1,30 & 4,00 & 0,880 & 16,08 \\
$\mathrm{KR}_{\mathrm{HCl}-3}$ & 1,28 & 4,60 & 0,795 & 15,00 \\
\hline
\end{tabular}

está situado entre os obtidos para caulinitas de alta ordem estrutural e alta pureza $(\mathrm{HI}>1)^{37}$ e que caulinitas com baixos valores de CTC apresentam altas cristalinidade e pureza. ${ }^{14}$ Os valores de CTC obtidos para as amostras sob ativação ácida são inferiores ao da amostra $\mathrm{KR}_{\text {natural }}$, devido ao ataque das hidroxilas da estrutura da caulinita.

$\mathrm{O}$ valor de $\mathrm{S}_{\mathrm{BET}}$ do rejeito de caulim apresentou pequeno aumento após tratamentos ácidos (Tabela 1). Este aspecto foi também verificado para outra amostra de caulim após tratamento com $\mathrm{HCl} 6 \mathrm{~mol}$ $\mathrm{L}^{-1}$ que aumentou de 17,0 para $18,9 \mathrm{~m}^{2} \mathrm{~g}^{-1} \cdot{ }^{39}$ Valores superiores de área específica podem ser obtidos quando caulinitas são submetidas a tratamentos térmicos $\left(\mathrm{T}>600^{\circ} \mathrm{C}\right)$ e mecânicos (moagem), seguidos de lixiviações ácidas. Nestas condições, a área específica dos materiais produzidos pode alcançar valores próximos de $533 \mathrm{~m}^{2} \mathrm{~g}^{-1} \cdot{ }^{38}$

\section{Distribuição do íon Pb (II) nas interfaces caulinitas-soluções}

As concentrações de equilíbrio (Ce) do íon $\mathrm{Pb}$ (II) em solução estão diretamente relacionadas com as medidas de $\mathrm{pH}$ obtidas na fase líquida. Nos testes de adsorção realizados os valores de $\mathrm{pH}_{\text {inicial }}$ medidos são inferiores a 6. Nestes valores de $\mathrm{pH}$ as frações de $\mathrm{Pb}$ (II) mais significativas são as de $\mathrm{Pb}^{2+}, \mathrm{PbOH}^{+} \mathrm{e} \mathrm{Pb}(\mathrm{OH})_{2}{ }^{39}$, as quais podem ser deduzidas a partir das reações de hidrólise (A e B) e substituindo-se as concentrações dos íons $\mathrm{H}^{+}$, correspondentes às medidas experimentais dos processos de adsorção, calcularam-se os valores das respectivas frações $\alpha_{\mathrm{Pb}}{ }^{2+}, \alpha_{\mathrm{PbOH}}{ }^{+}$e $\alpha_{\mathrm{Pb}(\mathrm{OH}) 2}$, usando as Equações 10 a 12:7-9

$\begin{array}{lll}\text { (A) } & \mathrm{Pb}^{2+}+\mathrm{H}_{2} \mathrm{O} \rightleftharpoons \mathrm{PbOH}^{+}+\mathrm{H}^{+} & \mathrm{K}_{1}=1,948 \times 10^{-8} \\ \text { (B) } \mathrm{Pb}^{2+}+2 \mathrm{H}_{2} \mathrm{O} \rightleftharpoons \mathrm{Pb}(\mathrm{OH})_{2}+2 \mathrm{H}^{+} & \mathrm{K}_{2}=7,58 \times 10^{-18}\end{array}$

$\alpha_{\mathrm{Pb}^{2+}}=\frac{\left[\mathrm{Pb}^{2+}\right]}{[\mathrm{Ce}]} \quad \therefore \quad \alpha_{\mathrm{Pb}^{2+}}=1+\frac{\left[\mathrm{H}^{+}\right]}{\mathrm{K}_{1}}+\frac{\left[\mathrm{H}^{+}\right]^{2}}{\mathrm{~K}_{2}}$

$\alpha_{\mathrm{PbOH}^{+}}=\frac{\left[\mathrm{PbOH}^{2+}\right]}{[\mathrm{Ce}]} \quad \therefore \quad \alpha_{\mathrm{PbOH}^{+}}=1+\frac{\mathrm{K}_{1}}{\left[\mathrm{H}^{+}\right]}+\frac{\mathrm{K}_{1}\left[\mathrm{H}^{+}\right]}{\mathrm{K}_{2}}$

$\alpha_{\mathrm{Pb}(\mathrm{OH})_{2}}=\frac{\left[\mathrm{Pb}(\mathrm{OH})_{2}\right]}{[\mathrm{Ce}]} \quad \therefore \quad \alpha_{\mathrm{Pb}(\mathrm{OH})_{2}}=1+\frac{\mathrm{K}_{2}}{\left[\mathrm{H}^{+}\right]^{2}}+\frac{\mathrm{K}_{2}}{\mathrm{~K}_{1}\left[\mathrm{H}^{+}\right]}$

$\mathrm{Na}$ fase sólida as concentrações das espécies de $\mathrm{Pb}(\mathrm{II})$ podem ser calculadas com base nas combinações de dados experimentais e de modelagens sobre reações de adsorção do $\mathrm{Pb}^{2+}$ nos sítios da caulinita. , $^{3,-9,40}$

Os resultados dos cálculos das frações molares das espécies de $\mathrm{Pb}(\mathrm{II})$, efetuados a partir das substituições das concentrações finais de íons $\mathrm{H}^{+}$medidas nos processos de adsorção nas Equações 10 a 12 , indicam que a espécie de $\mathrm{Pb}(\mathrm{II})$ predominante em solução é a de $\mathrm{Pb}^{2+}$ e a menos expressiva é a $\mathrm{Pb}(\mathrm{OH})_{2}$, como mostram os respectivos intervalos de valores das frações molares (Tabela 2).

Avaliando-se as concentrações de $\mathrm{H}^{+}, \mathrm{Pb}^{2+}, \mathrm{PbOH}^{+}$e $\mathrm{Pb}(\mathrm{OH})_{2}$, calculadas a partir das substituições das concentrações de Ce nas Equações 10 a 12, em relação às concentrações iniciais (Ci) de $\mathrm{Pb}$ (II) nos processos de adsorção a $30^{\circ} \mathrm{C}$ verifica-se que na amostra $\mathrm{KR}_{\text {natural }}$ 
Tabela 2. Intervalos de $\mathrm{pH}$ (inicial e final) e frações molares das espécies $\mathrm{Pb}^{2+}, \mathrm{PbOH}^{+}$e $\mathrm{Pb}(\mathrm{OH})_{2}$ dos processos de adsorção em $\mathrm{T}=303 \mathrm{~K}$

\begin{tabular}{|c|c|c|c|c|c|}
\hline Amostras & $\mathrm{pH}_{\text {inicial }}$ & $\mathrm{pH}_{\text {final }}$ & $\alpha \mathrm{Pb}^{2+}(\%)$ & $\alpha \mathrm{PbOH}^{+}(\%)$ & $\alpha \mathrm{Pb}(\mathrm{OH})_{2}(\%)$ \\
\hline $\mathrm{KR}_{\text {natural }}$ & $5,13-5,20$ & $4,85-3,84$ & $99,86-99,98$ & $1,4 \times 10^{-1}-1,3 \times 10^{-2}$ & $2,8 \times 10^{-3}-2,7 \times 10^{-4}$ \\
\hline $\mathrm{KR}_{\mathrm{H} 2 \mathrm{SO} 4-3}$ & $4,01-3,85$ & $3,86-3,58$ & $99,98-99,99$ & $1,4 \times 10^{-2}-0,74 \times 10^{-2}$ & $2,89 \times 10^{-4}-1,52 \times 10^{-4}$ \\
\hline $\mathrm{KR}_{\mathrm{HCl}-3}$ & $4,40-4,17$ & $4,04-4,24$ & $99,98-99,96$ & $2,15 \times 10^{-2}-2,7 \times 10^{-2}$ & $4,4 \times 10^{-4}-6,9 \times 10^{-4}$ \\
\hline
\end{tabular}

(Figura 1) as concentrações de $\mathrm{H}^{+}$e as das três espécies de $\mathrm{Pb}(\mathrm{II})$ aumentam gradualmente conforme aumento de $\mathrm{Ci}$.

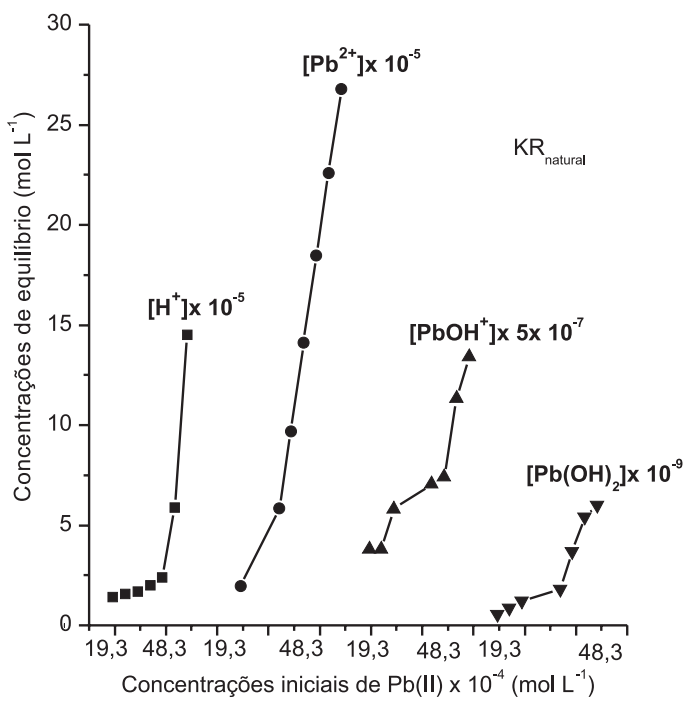

Figura 1. Distribuição das concentrações de equilíbrio de $\mathrm{H}^{+}, \mathrm{Pb}^{2+}, \mathrm{PbOH}^{+}$ e $\mathrm{Pb}(\mathrm{OH})_{2}$ em relação às concentrações nos processos de adsorção do $\mathrm{Pb}(\mathrm{II})$ na amostra $K R_{\text {natural }}$

Nos adsorventes $\mathrm{KR}_{\mathrm{H} 2 \mathrm{SO}-3}$ e $\mathrm{KR}_{\mathrm{HCl}-3}$ (Figuras 2 e 3 ) a concentração de $\mathrm{Pb}^{2+}$ apresenta a mesma distribuição observada em $\mathrm{KR}_{\text {natural }}$, mas há divergência em relação ao padrão de distribuição da concentração de $\mathrm{H}^{+}$dos três adsorventes. Durante o processo de adsorção em $\mathrm{KR}_{\mathrm{H} 2 \mathrm{SO}-3}$ a concentração de $\mathrm{H}^{+}$é muito variável e em $\mathrm{KR}_{\mathrm{HCl}-3}$ a concentração de $\mathrm{H}^{+}$decresce gradualmente com o aumento de $\mathrm{Ci}$. Este aspecto pode ser atribuído às reações de protonação e desprotonação durante os processos de adsorção.

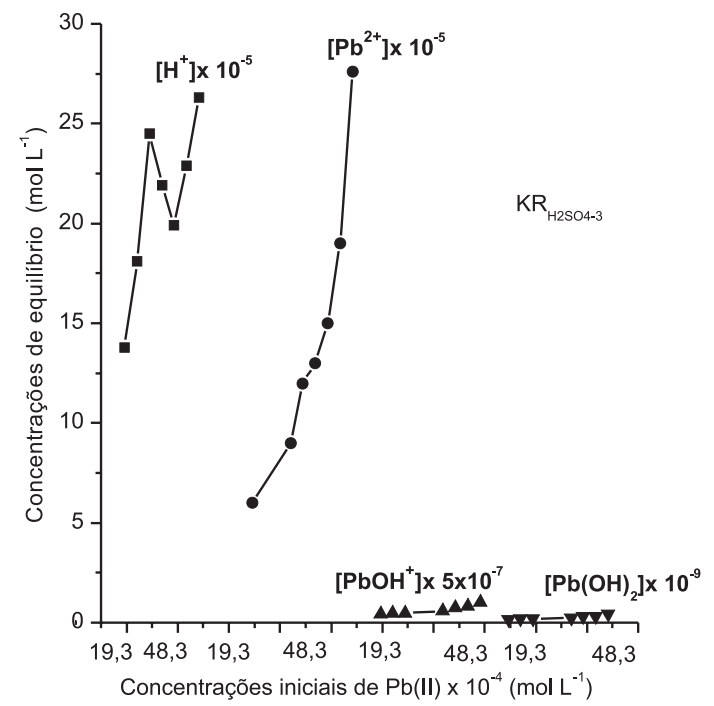

Figura 2. Distribuição das concentrações de equilíbrio de $\mathrm{H}^{+}, \mathrm{Pb}^{2+}, \mathrm{PbOH}^{+}$ e $\mathrm{Pb}(\mathrm{OH})_{2}$ em relação às concentraçães nos processos de adsorção do $\mathrm{Pb}(\mathrm{II})$ na amostra $K_{\text {H2SO4-3 }}$

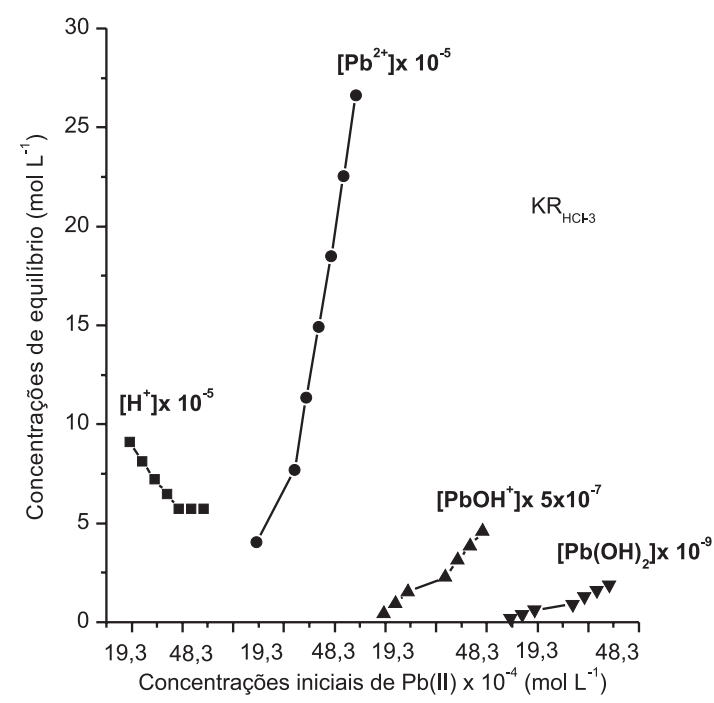

Figura 3. Distribuição das concentrações de equilíbrio de $\mathrm{H}^{+}, \mathrm{Pb}^{2+}, \mathrm{PbOH}^{+}$ e $\mathrm{Pb}(\mathrm{OH})_{2}$ em relação às concentrações nos processos de adsorção do $\mathrm{Pb}(\mathrm{II})$ na amostra $\mathrm{KR}_{\mathrm{HCl}-3}$

Equilíbrio de adsorção do $\mathrm{Pb}(\mathrm{II})$ nas interfaces caulinitassoluções

\section{Quantidades adsorvidas do $\mathrm{Pb}(\mathrm{II})$}

$\mathrm{O}$ valor médio de qe obtido no rejeito de caulim sem tratamento foi $4,14 \mathrm{mg} \mathrm{g}^{-1}$ e encontra-se no intervalo de qe obtido na adsorção do $\mathrm{Pb}$ (II) em amostra de caulinita da Companhia de Caulim, Fujian. ${ }^{16}$ Em termos da quantidade de $\mathrm{Pb}$ (II) adsorvida (qe) em relação às concentrações iniciais (Figura 4) verificou-se aumento de qe em direção às maiores concentrações iniciais e que os tratamentos com soluções ácidas efetuados no rejeito de caulim não contribuíram para o aumento de qe médio. As diferenças de qe nos adsorventes ressaltam-se apenas nos pontos iniciais dos processos de adsorção. Nos pontos finais (concentrações iniciais mais elevadas) os valores de qe tendem a se aproximar, especialmente os de $\mathrm{KR}_{\text {natural }}$ e $\mathrm{KR}_{\mathrm{H} 2 \mathrm{SO}-3.3}$. Nos três processos de adsorção os valores de qe médios são bem próximos entre si, sendo o de $\mathrm{KR}_{\text {natural }}$ apenas cerca de $1 \%$ superior aos qe médios dos outros adsorventes. Este aspecto não se justifica pelos valores de $S_{\text {BET }}$ que são menores em $\mathrm{KR}_{\text {natural }}$, mas podem ser atribuídos em princípio ao maior valor de CTC de $\mathrm{KR}_{\text {natural }}$ (Tabela 1). Seria esperado ocorrer aumento de qe mais próximo do valor obtido na adsorção do $\mathrm{Pb}$ (II) em caulinita tratada com solução de $\mathrm{H}_{2} \mathrm{SO}_{4}$, que apresentou qe em torno de $3,32 \%$ superior ao da caulinita sem tratamento. ${ }^{32}$

$\mathrm{Na}$ avaliação de qe em relação aos valores de $\mathrm{pH}$ final dos processos de adsorção (Figura 5) verificou-se aumento gradual e contínuo de $\mathrm{Pb}$ (II) adsorvido com o decréscimo de $\mathrm{pH}$ em $\mathrm{KR}_{\text {natural }}$ e com o aumento de $\mathrm{pH}$ em $\mathrm{KR}_{\mathrm{HCl}-3}$. Em KR $\mathrm{KR}_{\mathrm{H} 2 \mathrm{SO} 4-3}$ a distribuição dos valores de qe em relação ao $\mathrm{pH}$ é descontínua, devido provavelmente à variação de cargas ocorrida durante o processo de adsorção.

$\mathrm{O}$ menor valor de qe médio verificado em $\mathrm{KR}_{\mathrm{H} 2 \mathrm{SO}-3}$ pode ser resultante da condição mais ácida do processo de adsorção, que deve ter propiciado aumento de carga positiva e, consequentemente, repulsão eletrostática entre $\mathrm{Pb}^{2+}$ em grupos superficiais positivamente 


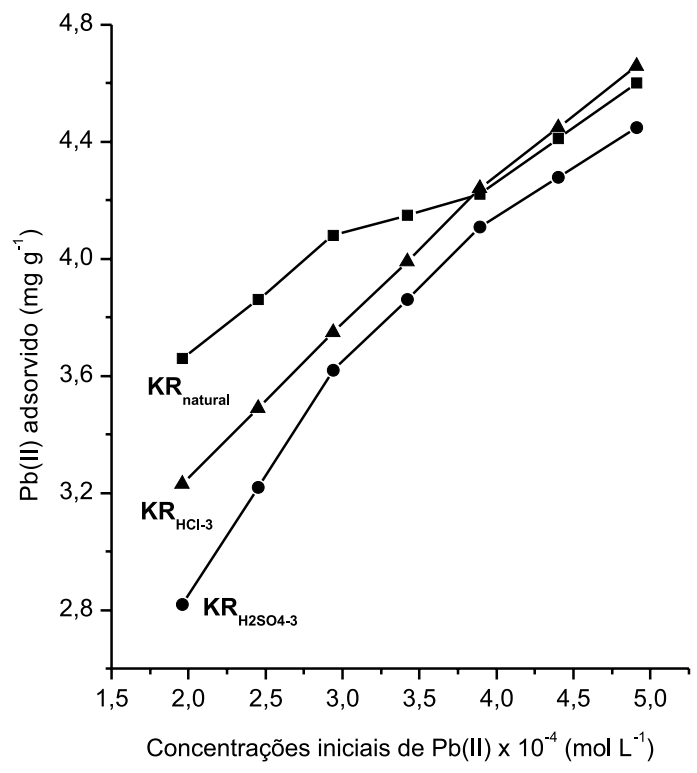

Figura 4. Quantidade de $\mathrm{Pb}(\mathrm{II})$ adsorvido em relação às concentrações iniciais usadas nos processos de adsorção nas amostras $K R_{\text {natural }} K R_{H 2 S O 4-3}$ e KR $R_{H C l-3}$

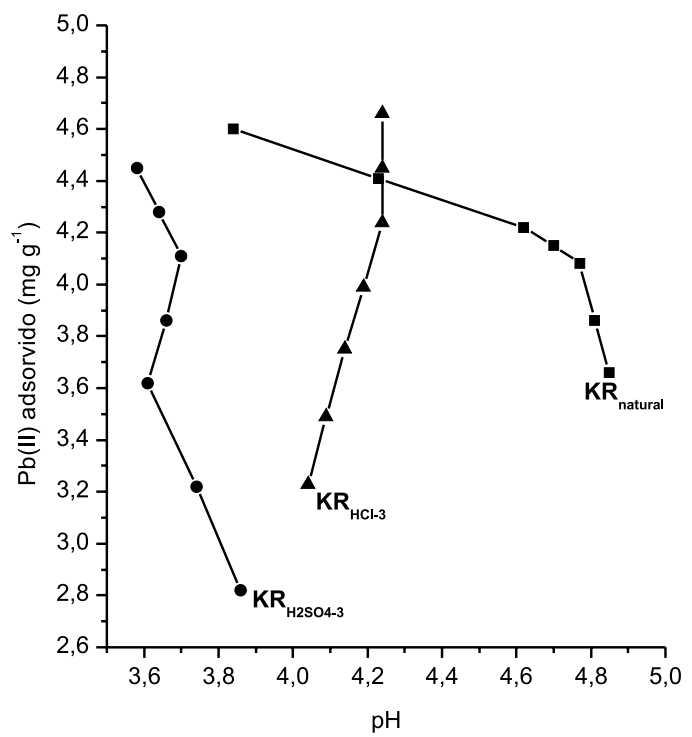

Figura 5. Variabilidade da quantidade de $P b(I I)$ adsorvido em relação ao pH final dos processos de adsorção nas amostras $K_{\text {natural }} K_{\mathrm{H}_{2504-3}}$ e KR $R_{\mathrm{HCl}-3}$

carregados da caulinita, conforme pode ser deduzido a partir de outros experimentos de adsorção de $\mathrm{Pb}(\mathrm{II})$ em caulinita. ${ }^{4}$

Química superficial do $\mathrm{Pb}(I I)$ nas interfaces caulinitas-soluções

As justificativas sobre as variações das quantidades de $\mathrm{Pb}$ (II) adsorvidas em relação ao $\mathrm{pH}$ nas interfaces caulinitas-soluções estão baseadas em modelos de formação de complexos superficiais usados para explicar a adsorção de cátions metálicos $\mathrm{M}^{2+}$ nos sítios de adsorção da caulinita. $\mathrm{O} \mathrm{Pb}^{2+}$, assim como outros cátions metálicos $\mathrm{M}^{2+}$, pode ser adsorvido no sitio de carga negativa permanente na face tetraédrica, indicado como $\mathrm{X}^{-}$ou $\mathrm{SiO}^{-}$, e nos sítios de cargas variáveis nas bordas dos cristais e na face octaédrica da caulinita, dependentes do $\mathrm{pH} .{ }^{3,7-9,40}$

Nos sítios de carga negativa permanente (face tetraédrica) os modelos de formação de complexos superficiais são indicados pelas reações: ${ }^{7,8}$

$\mathrm{SiO}^{-}+\mathrm{M}^{2+} \rightleftharpoons(\mathrm{SiO})_{2} \mathrm{M} \quad \mathrm{K}=3,55 \times 10^{3}$
Nos sítios de cargas variáveis, dependentes do $\mathrm{pH}$ (bordas e face octaédrica) as interações superficiais são explicadas com base nas reações: ${ }^{3,38}$

1) Protonação e desprotonação do grupo aluminol nas bordas
$\Xi \mathrm{AlOH}+\mathrm{H}^{+} \rightleftharpoons \Xi \mathrm{Al}(\mathrm{OH})_{2}{ }^{+}$
$\log \mathrm{K}_{\left(25^{\circ} \mathrm{C}\right)}=4,80$
$\Xi \mathrm{AlOH} \rightleftharpoons \Xi \mathrm{AlO}^{-}+\mathrm{H}^{+}$
$\log \mathrm{K}_{\left(25^{\circ} \mathrm{C}\right)}=-6,10$

2) Desprotonação do grupo silanol nas bordas

$\Xi \mathrm{SiOH} \rightleftharpoons \Xi \mathrm{SiO}^{-}+\mathrm{H}^{+} \quad \log \mathrm{K}_{\left(25^{\circ} \mathrm{C}\right)}=-7,70$

Considerando que os processos de adsorção do $\mathrm{Pb}(\mathrm{II})$ neste trabalho ocorreram em $\mathrm{pH}<5,2$ e que o intervalo de $\mathrm{pH}$ final do processo adsorção em $\mathrm{KR}_{\mathrm{H} 2 \mathrm{SO} 4-3}$ é menor do que os obtidos em $\mathrm{KR}_{\text {natural }}$ e $\mathrm{KR}_{\mathrm{HCl}-3 \text {, }}$ então pode-se assumir que o $\mathrm{Pb}$ (II) é adsorvido especialmente no sítio de carga negativa permanente da caulinita e que nos sítios de cargas dependentes do $\mathrm{pH}$ o $\mathrm{KR}_{\mathrm{H} 2 \mathrm{SO} \text {-3 }}$ é o adsorvente que apresenta repulsão eletrostática mais elevada entre as espécies positivamente carregadas de $\mathrm{Pb}(\mathrm{II})$ e o grupo aluminol protonado.

Isotermas de adsorção do Pb(II) nas interfaces caulinitas-soluções

Os modelos de isotermas de Langmuir e Freundlich foram adequados no ajuste dos dados experimentais de adsorção do $\mathrm{Pb}$ (II) em rejeito de caulim (Tabela 3). De acordo com os valores de erros obtidos, os melhores ajustes pelos dois modelos de isotermas foram verificados na seguinte ordem: $\mathrm{KR}_{\mathrm{H} 2 \mathrm{SO} 4-3}>\mathrm{KR}_{\text {natural }}>\mathrm{KR}_{\mathrm{HCl}-3}$. Os valores da constante $\mathrm{n}$ de Freundlich superiores à unidade, assim como os obtidos a partir de outras caulinitas, sugerem que os processos de adsorção do $\mathrm{Pb}(\mathrm{II})$ neste estudo são favoráveis.

Tabela 3. Parâmetros das isotermas de adsorção do íon $\mathrm{Pb}$ (II) nas amostras estudadas

\begin{tabular}{cccc}
\hline Isotermas & $\mathrm{KR}_{\text {natural }}$ & $\mathrm{KR}_{\mathrm{H} 2 \mathrm{SO} 4-3}$ & $\mathrm{KR}_{\mathrm{HCl}-3}$ \\
\hline Langmuir & & & \\
$\mathrm{q}_{\text {máx }}\left(\mathrm{mg} \mathrm{g}^{-1}\right)$ & $4,39 \pm 0,11$ & $5,30 \pm 0,07$ & $4,83 \pm 0,20$ \\
$\mathrm{~K}_{\mathrm{L}}\left(\mathrm{L} \mathrm{g}^{-1}\right)$ & $1,02 \pm 0,33$ & $0,09 \pm 0,01$ & $0,19 \pm 0,05$ \\
$\mathrm{R}^{2}$ & 0,7144 & 0,9949 & 0,8630 \\
Erro $(\%)$ & 4,01 & 1,80 & 5,13 \\
\hline Freundlich & & & \\
$\mathrm{K}_{\mathrm{F}}\left(\mathrm{L} \mathrm{g}^{-1}\right)$ & $3,18 \pm 0,12$ & $1,38 \pm 0,07$ & $2,01 \pm 0,11$ \\
$\mathrm{n}_{\mathrm{F}}$ & $11,94 \pm 1,59$ & $3,41 \pm 0,17$ & $4,87 \pm 0,36$ \\
$\mathrm{R}^{2}$ & 0,9234 & 0,9888 & 0,9752 \\
Erro $(\%)$ & 2,13 & 1,78 & 2,19 \\
\hline
\end{tabular}

Comparando-se os dados de $\mathrm{q}_{\text {máx }}$ obtidos na adsorção do $\mathrm{Pb}$ (II) neste estudo com os obtidos em outros estudos de adsorção do $\mathrm{Pb}$ (II) em padrões de caulinita e montmorilonita (KGa-b e padrão Swy-2, respectivamente) e em outras caulinitas(Tabela 4), verifica-se que o

Tabela 4. Capacidade máxima de adsorção ( $q_{\text {máx. }}$ obtidos a partir da isoterma de Langmuir) para adsorção de $\mathrm{Pb}(\mathrm{II})$ em caulinita e montmorilonita de diferentes origens

\begin{tabular}{lcc}
\hline Adsorvente & $\mathrm{q}_{\text {máx }}\left(\mathrm{mg} \mathrm{g}^{-1}\right)$ & Ref. \\
\hline Caulim Fujian & 4,73 & 16 \\
Caulinita KGa-1b & 11,52 & 31 \\
Montmorilonita Swy-2 & 31,05 & 31 \\
Caulinita Jordânia & 13,32 & 33 \\
Caulinita Jordânia com $\mathrm{H}_{2} \mathrm{SO}_{4} 5 \mathrm{~mol} \mathrm{~L}^{-1}$ & 51,59 & 33 \\
\hline
\end{tabular}


Tabela 5. Coeficiente de separação das amostras estudadas em quatro temperaturas (K)

\begin{tabular}{|c|c|c|c|c|c|c|c|c|c|c|c|}
\hline \multicolumn{12}{|c|}{ Coeficiente de separação $R_{L}$} \\
\hline \multicolumn{4}{|c|}{$\mathrm{KR}_{\text {natural }}$} & \multicolumn{4}{|c|}{$\mathrm{KR}_{\mathrm{H} 2 \mathrm{SO}-3}$} & \multicolumn{4}{|c|}{$\mathrm{KR}_{\mathrm{HCl}-3}$} \\
\hline 303 & 313 & 318 & 323 & 303 & 313 & 318 & 323 & 303 & 313 & 318 & 323 \\
\hline 0,377 & 0,835 & 0,837 & 0,839 & 0,486 & 0,690 & 0,745 & 0,821 & 0,896 & 0,891 & 0,835 & 0,755 \\
\hline 0,170 & 0,800 & 0,795 & 0,790 & 0,387 & 0,607 & 0,68 & 0,777 & 0,810 & 0,866 & 0,794 & 0,694 \\
\hline 0,110 & 0,767 & 0,756 & 0,745 & 0,322 & 0,542 & 0,626 & 0,738 & 0,754 & 0,842 & 0,758 & 0,642 \\
\hline 0,078 & 0,725 & 0,717 & 0,710 & 0,268 & 0,503 & 0,586 & 0,703 & 0,700 & 0,816 & 0,721 & 0,598 \\
\hline 0,061 & 0,687 & 0,683 & 0,679 & 0,229 & 0,469 & 0,552 & 0,671 & 0,653 & 0,791 & 0,688 & 0,559 \\
\hline 0,050 & 0,679 & 0,664 & 0,649 & 0,196 & 0,471 & 0,553 & 0,672 & 0,606 & 0,789 & 0,686 & 0,556 \\
\hline 0,043 & 0,672 & 0,647 & 0,623 & 0,171 & 0,473 & 0,554 & 0,672 & 0,566 & 0,787 & 0,684 & 0,553 \\
\hline
\end{tabular}

$\mathrm{q}_{\text {máx }}$ do rejeito de caulim sem tratamento $\left(\mathrm{KR}_{\text {natural }}\right)$ é muito próximo ao da caulinita de Fujian ${ }^{16}$ e inferior aos dos padrões de caulinita, caulinita da Jordânia natural e tratada com $\mathrm{H}_{2} \mathrm{SO}_{4} 5 \mathrm{~mol} \mathrm{~L}^{-1} \mathrm{e}$ ao da montmorilonita. ${ }^{31,33}$

A grande diferença entre os $\mathrm{q}_{\text {máx }}$ obtidos para a adsorção do $\mathrm{Pb}(\mathrm{II})$ em $\mathrm{KR}_{\mathrm{H} 2 \mathrm{SO} 4-3}$ e caulinita da Jordânia tratada com $\mathrm{H}_{2} \mathrm{SO}_{4} 5 \mathrm{~mol} \mathrm{~L}^{-1}$ pode ser atribuída aos seus valores de CTC, que são 7,42 e 4,0 $\mathrm{cmol}_{\mathrm{c}} \mathrm{kg}^{-1}$, respectivamente. ${ }^{33}$ Não foi possível justificar esta diferença com base nos valores de área específica, porque não se tem informação do valor para a amostra sob ativação ácida e sim para caulinita natural, que apresenta $\mathrm{S}=16,9 \mathrm{~m}^{2} \mathrm{~g}^{-1}$ e $\mathrm{CTC}=8,97 \mathrm{cmol}_{\mathrm{c}} \mathrm{kg}^{-1} \cdot{ }^{33}$

\section{Abordagem termodinâmica dos processos de adsorção}

Coeficientes de separação, constantes de equilíbrio e variação de energia livre

$\mathrm{Na}$ avaliação dos coeficientes de separação, em $\mathrm{T}=303,313$, 318 e $323 \mathrm{~K}$, em relação às concentrações iniciais dos processos de adsorção, verificou-se que os valores de $R_{L}$ em $K_{\text {natural }}$ são inferiores aos obtidos para $\mathrm{KR}_{\mathrm{H} 2 \mathrm{SO}-3}$ e $\mathrm{KR}_{\mathrm{HCl}-3}$ nas quatro temperaturas (Tabela 5). Estes dados de $R_{L}$ indicam que a adsorção do $\mathrm{Pb}$ (II) em $\mathrm{KR}_{\text {natural }}$ é mais viável do que $\mathrm{KR}_{\mathrm{H} 2 \mathrm{SO}-3}$ e $\mathrm{KR}_{\mathrm{HCl}-3}$.

As evidências indicadas acima sobre as viabilidades dos processos de adsorção ressaltam-se quando os valores dos coeficientes de separação e variação são avaliados em relação às concentrações iniciais (Figura 6). Observa-se com base nos valores dos coeficientes de separação e variação em $\mathrm{T}=303 \mathrm{~K}$ que a viabilidade de adsorção do $\mathrm{Pb}$ (II) é maior em $\mathrm{KR}_{\text {natural }}$ e menor em $\mathrm{KR}_{\mathrm{HCl}-3}$ e que nos três processos de adsorção as reações de adsorção se tornam mais viáveis em direção às maiores concentrações iniciais.

\section{Variações de energia livre de Gibbs, entalpia e entropia}

Com base nos valores das constantes $\mathrm{K}_{\mathrm{L}}$ calculados a partir da equação linearizada de Langmuir foram construídos os gráficos $\ln \mathrm{K}_{\mathrm{L}}$ versus 1/T (Figura 3S, material suplementar), que fornecem relações lineares, com coeficientes angulares (iguais a $-\Delta H^{\circ} / R$ ) e coeficientes lineares $\left(\Delta \mathrm{S}^{\circ} / \mathrm{R}\right)$. Os resultados dos cálculos efetuados para determinação de $\Delta \mathrm{H}^{\circ}$ e $\Delta \mathrm{S}^{\circ}$, assim como os de $\Delta \mathrm{G}^{\circ}$ calculados

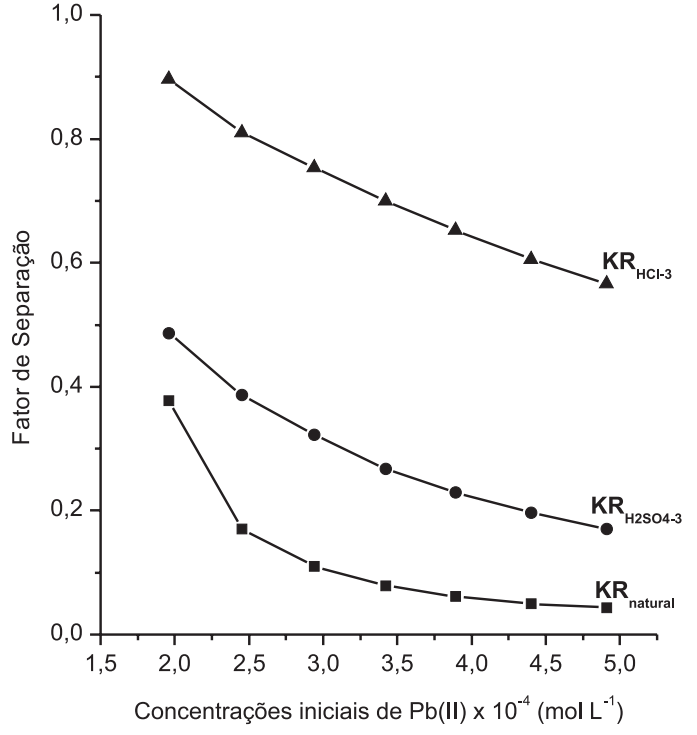

Figura 6. Distribuição do fator de separação $R_{L}$ em relação às concentrações iniciais dos processos de adsorção do $\mathrm{Pb}(\mathrm{II})$ nas amostras $K R_{\text {natural }}, K R_{H 2 S O 4-3}$ e KR $R_{H C l-3}$

pela Equação 7, correspondentes aos processos de adsorção, estão indicados na Tabela 6 .

Os valores das variações de energia livre, entalpia e entropia $\Delta \mathrm{S}^{\circ}$ são negativos, assim como outros processos de adsorção do $\mathrm{Pb}$ (II) em caulinitas. ${ }^{17,27}$ Estes dados indicam que os processos de adsorção são espontâneos, exotérmicos e acompanhados por decréscimo da entropia. De acordo com revisão teórica sobre a Segunda Lei da Termodinâmica, ${ }^{41}$ os processos espontâneos que resultam na diminuição da entropia do sistema são sempre exotérmicos. A variação de entropia (variação de desordem) de um processo químico afeta a espontaneidade deste processo. Quanto mais desordenado ou aleatório um sistema, maior a sua variação de entropia e menor a sua espontaneidade. ${ }^{39}$

Entre os processos de adsorção do $\mathrm{Pb}$ (II) nas caulinitas deste estudo o mais ordenado (o que apresenta menor variação de entropia,

Tabela 6. Parâmetros termodinâmicos do processo de adsorção do íon $\mathrm{Pb}(\mathrm{II})$ sobre rejeito de caulim natural e modificado

\begin{tabular}{|c|c|c|c|c|c|c|}
\hline \multirow{2}{*}{ Adsorvente } & \multirow{2}{*}{$\Delta \mathrm{H}^{\mathrm{o}}\left(\mathrm{kJ} \mathrm{mol}^{-1}\right)$} & \multirow{2}{*}{$\Delta \mathrm{S}^{\mathrm{o}}\left(\mathrm{J} \mathrm{mol}^{-1} \mathrm{~K}^{-1}\right)$} & \multicolumn{4}{|c|}{$\Delta \mathrm{G}^{\mathrm{o}}\left(\mathrm{J} \mathrm{mol}^{-1} \mathrm{~K}^{-1}\right)$} \\
\hline & & & $303 \mathrm{~K}$ & $313 \mathrm{~K}$ & $318 \mathrm{~K}$ & $323 \mathrm{~K}$ \\
\hline $\mathrm{KR}_{\text {natural }}$ & $-110,30$ & $-290,91$ & $-28,55$ & $-22,25$ & $-22,63$ & $-22,99$ \\
\hline $\mathrm{KR}_{\mathrm{H} 2 \mathrm{SO} 4-3}$ & $-50,69$ & $-85,30$ & $-24,62$ & $-24,28$ & $-23,85$ & $-22,85$ \\
\hline $\mathrm{KR}_{\mathrm{HCl}-3}$ & $-56,59$ & $-106,58$ & $-25,88$ & $-20,54$ & $-22,31$ & $-24,09$ \\
\hline
\end{tabular}


durante a evolução das reações) é o da adsorção em $\mathrm{KR}_{\text {natural }}$, conforme pode ser deduzido pelos valores mais negativos de $\Delta \mathrm{S}^{\circ}$ (Tabela 6).

De acordo com os valores absolutos de $\Delta \mathrm{H}^{\circ}$ ocorre fisissorção se $\Delta \mathrm{H}^{\circ}$ dos processos de adsorção alcançam valores inferiores a $80 \mathrm{KJ}$ mol $^{-1}$ e quimissorção se os valores $\Delta \mathrm{H}^{\circ}$ estão compreendidos entre 80 a $420 \mathrm{KJ} \mathrm{mol}^{-1} .{ }^{5}$ Portanto, com base nestas informações é possível sugerir que o valor absoluto obtido para a variação de entalpia no processo de adsorção em $\mathrm{KR}_{\mathrm{H} 2 \mathrm{SO4}-3}$ está compreendido entre os esperados para fisissorção e os obtidos em $\mathrm{KR}_{\text {natural }}$ e $\mathrm{KR}_{\mathrm{HCl}-3}$ estão compreendidos entre os esperados para quimissorção.

\section{CONCLUSÕES}

Nos processos de adsorção do $\mathrm{Pb}(\mathrm{II})$ em amostras de rejeito de caulim, natural ( $\left.\mathrm{KR}_{\text {natural }}\right)$ e tratadas com soluções de $\mathrm{H}_{2} \mathrm{SO}_{4}$ e $\mathrm{HCl} 3$ mol L-1 $\left(\mathrm{KR}_{\mathrm{H} 2 \mathrm{SO}-3}\right.$ e $\mathrm{KR}_{\mathrm{HCl}-3}$, respectivamente), constituídas predominantemente por caulinita chegou-se às conclusões a seguir.

Nos intervalos naturais de $\mathrm{pH}$ dos processos de adsorção $(\mathrm{pH}<$ 5,2 ) as espécies químicas de $\mathrm{Pb}$ (II) em equilíbrio ocorrem na seguinte ordem de concentração: $\left[\mathrm{Pb}^{2+}\right]>\left[\mathrm{PbOH}^{+}\right]>\left[\mathrm{Pb}(\mathrm{OH})_{2}\right]$.

A eficiência dos processos de adsorção, avaliada em termos da quantidade de $\mathrm{Pb}$ (II) adsorvida (qe) em relação às concentrações iniciais e $\mathrm{pH}$ de equilíbrio, segue a ordem de adsorção em $\mathrm{KR}_{\text {natural }}$ $>\mathrm{KR}_{\mathrm{HCl}-3}>\mathrm{KR}_{\mathrm{H} 2 \mathrm{SO} 4-3}$.

Com base nos valores dos coeficientes de separação e variação de energia livre (em T = 303, 313, 318 e $323 \mathrm{~K}$ ) foi verificado que todos os processos de adsorção são viáveis, espontâneos. Os resultados obtidos para as variações de entalpia $\left(\Delta \mathrm{H}^{\circ}\right)$ e entropia $\left(\Delta \mathrm{S}^{\circ}\right)$ indicaram que os processos de adsorção são exotérmicos e ocorrem com decréscimo de entropia.

Os parâmetros mais importantes na distinção dos processos de adsorção do $\mathrm{Pb}$ (II) foram $\mathrm{R}_{\mathrm{L}}, \Delta \mathrm{H}^{\circ}$ e $\Delta \mathrm{S}^{\circ}$, que permitiram indicar a adsorção em $\mathrm{KR}_{\text {natural }}$ como a mais viável, do tipo química (quimissorção) e a mais ordenada.

\section{MATERIAL SUPLEMENTAR}

A Figura $1 \mathrm{~S}$ referente aos difratogramas de raios $\mathrm{X}$ do rejeito de caulim natural e rejeito de caulim modificados, assim como a Figura $2 \mathrm{~S}$ referente às micrografias e a Figura $3 \mathrm{~S}$ relativa aos cálculos dos parâmetros termodinâmicos estão disponíveis em http://quimicanova. sbq.org.br na forma de arquivo PDF, com acesso livre.

\section{AGRADECIMENTOS}

Ao CNPq pelos recursos financeiros do projeto "Dos Minerais aos Novos Materiais (Convênio 2271-FAPESPA(PRONEX)/UFPA/ FADESP/SEDECT) e pela concessão de bolsas IC e de produtividade em pesquisa. À Vale pelos recursos financeiros do projeto (Edital 001-2010 FAPEMIG/FAPESP/FAPESPA/VALE S.A).

\section{REFERÊNCIAS}

1. Fritzen, M. B.; Souza, A. J.; Silva, T. A. G.; Souza, L.; Nome, R. A.; Fiedler, H. D.; Nome, F.; J. Colloid Interface Sci. 2006, 296, 465.

2. Li, Z.; Gallus, L.; Appl. Clay Sci. 2007, 35, 250.

3. Brady, P. V.; Randall, T. C.; Nagy, K. L.; J. Colloid Interface Sci. 1996, $183,356$.

4. Sari, A.; Tuzen, M.; Citak, D.; Soylak, M.; J. Hazard. Mater. 2007, 149, 283.
5. Sölener, M.; Tunali, S.; Özcan, A. S.; Özcan, A.; Gedikbey, T.; Desalination 2008, 223, 308.

6. Tertre, E.; Castet, S.; Berger, G.; Loubet, M.; Giffaut, E.; Geochim. Cosmochim. Acta 2006, 70, 4579.

7. Angove, M. J.; Johnson, B. B.; Wells, J. D.; Colloid Surf., A 1997, 126, 137.

8. Angove, M. J.; Johnson, B. B.; Wells, J. D.; J. Colloid Interface Sci. 1998, 204, 93.

9. Ikhsan, J.; Johnson, B. B.; Wells, J. D.; Angove, M. J.; J. Colloid Interface Sci. 2004, 273, 6.

10. Gasselin, R. E.; Smith, R. P.; Hodge, H. C.; Chemical Toxicology of Commercial Products, Williams and Wilkins: London, 1984.

11. Namasivayam, K. R.; Water Res. 1993, 32, 969.

12. Koller, K.; Bronw, T.; Spurgeon, A.; Levy, L.; Environ. Health Perspect. 2004, 112, 987.

13. Zanini, C. I. C. B.; Figueiredo, F. C. A.; Carvalho, W. A.; Quim. Nova 2009, 32, 2318.

14. Coles, C. A. E.; Yong, R. N.; Appl. Clay Sci. 2002, 22, 39.

15. Adebowale, K. O.; Unuabonah, E. I.; Olu-Owolabi, B. I.; Chem. Eng. J. 2008, 136, 99.

16. Jiang, M.-Q.; Wang, Q.-P.; Jin, X.-y.; Chen, Z.-1.; J. Hazard. Mater. 2009, 170, 332

17. http://www.dnpm.gov.br, acessada em Maio 2011.

18. Maia, A. A. B.; Angélica, R. S.; Neves, R. F.; Cerâmica 2008, 54, 345.

19. Barata, M. S.; Angélica, R. S.; Pollmann, H.; Costa, M. L.; Eur. J. Mineral. 2005, 17, 10

20. Maia, A. A. B.; Saldanha, E.; Angélica, R. S.; Souza, C. A. G.; Neves, R. F.; Cerâmica 2007, 53, 319.

21. Pinheiro, M. H. T.; Tese de Doutorado, Universidade Federal do Pará, Brasil, 2011.

22. Brunauer, S.; Emmett, P. H.; Teller, E.; J. Am. Chem. Soc. 1938, 60, 309.

23. Cimino, G.; Water Res. 2000, 34, 2955.

24. Allen, S. J.; Mckay, G.; Porter, J. F.; J .Colloid Interface Sci. 2004, 280, 322.

25. Langmuir, I.; J. Am. Chem. Soc. 1918, 40, 1361.

26. Singh, T. S.; Pant, K. K.; Sep. Purif. Technol. 2004, 36, 139.

27. Freundlich, H. M. F. Z.; Physico Chemical 1906, 57, 380.

28. Kumar, K. V.; Sivanesan, S.; J. Hazard. Mater. B 2005, 126, 198.

29. Karadac, D. A.; Koc, Y.; Turan, M.; Ozturk M.; J. Hazard. Mater. 2007, 144,432

30. Vaghetti, J. C. P.; Lima, E. C.; Royer, B.; Cunha, B. M. da; Cardoso, N. F.; Brasil, J. L.; Dias, S. L. P.; J. Hazard. Mater. 2009, 162, 270.

31. Gupta, S. S.; Bhattacharyya, K. G.; Appl. Clay Sci. 2005, 30, 199.

32. Gupta, S. S.; Bhattacharyya, K. G.; J. Environ. Management 2008, 87, 46.

33. Al-Harahsheh, M.; Shawabkeh, R.; Al-Harahsheh, A.; Tarawneh, K.; Batiha, M. M.; Appl. Surf. Sci. 2009, 255, 8098.

34. Liu, Y.; Xu, H.; Biochem. Eng. J. 2007, 35, 174.

35. Liu, Y.; Liu, Y.-J.; Sep. Purif. Technol. 2008, 61, 229.

36. Makó, E.; Senkar, Z.; Kristóf, J.; Vágvölgvi, V.; J. Colloid Interface Sci. 2006, 294, 362.

37. Coelho, A. C. C.; Santos, P. S.; Abstracts of the $12^{\text {th }}$ International Clay Conference, Bahia Blanca, Argentina, 2001.

38. Cristóbal, A. G. S.; Castelló, R.; Luengo, M. A. M.; Vizcayno, C.; Mater. Res. Bull. 2009, 44, 2103.

39. Bradl, H. B.; J. Colloid Interface Sci. 2004, 277, 1.

40. Schroth, B. K.; Sposito, G.; Environ. Sci. Technol. 1998, 32, 1404

41. Brown, T. L.; LeMay Jr., H. E.; Bursten, B. E.; Burdge, J. R.; Química A Ciência Central, $9^{\mathrm{a}}$ ed., Pearson Prentice Hall: São Paulo, 2007. 
Marta Helena Tavares Pinheiro*, Vanda Porpino Lemos e Kelly das G. Fernandes Dantas

Faculdade de Química, Instituto de Ciências Exatas e Naturais, Universidade Federal do Pará, Cidade Universitária Prof. José Silveira Netto, Setor Básico, Rua Augusto Corrêa, 1, 66075-110 Belém - PA, Brasil

Taynara Lima Valentim

Faculdade de Engenharia Química, Instituto Tecnológico, Universidade Federal do Pará, Cidade Universitária Prof. José Silveira Netto, Setor Profissional, Rua Augusto Corrêa, 1, 66075-110 Belém - PA, Brasil
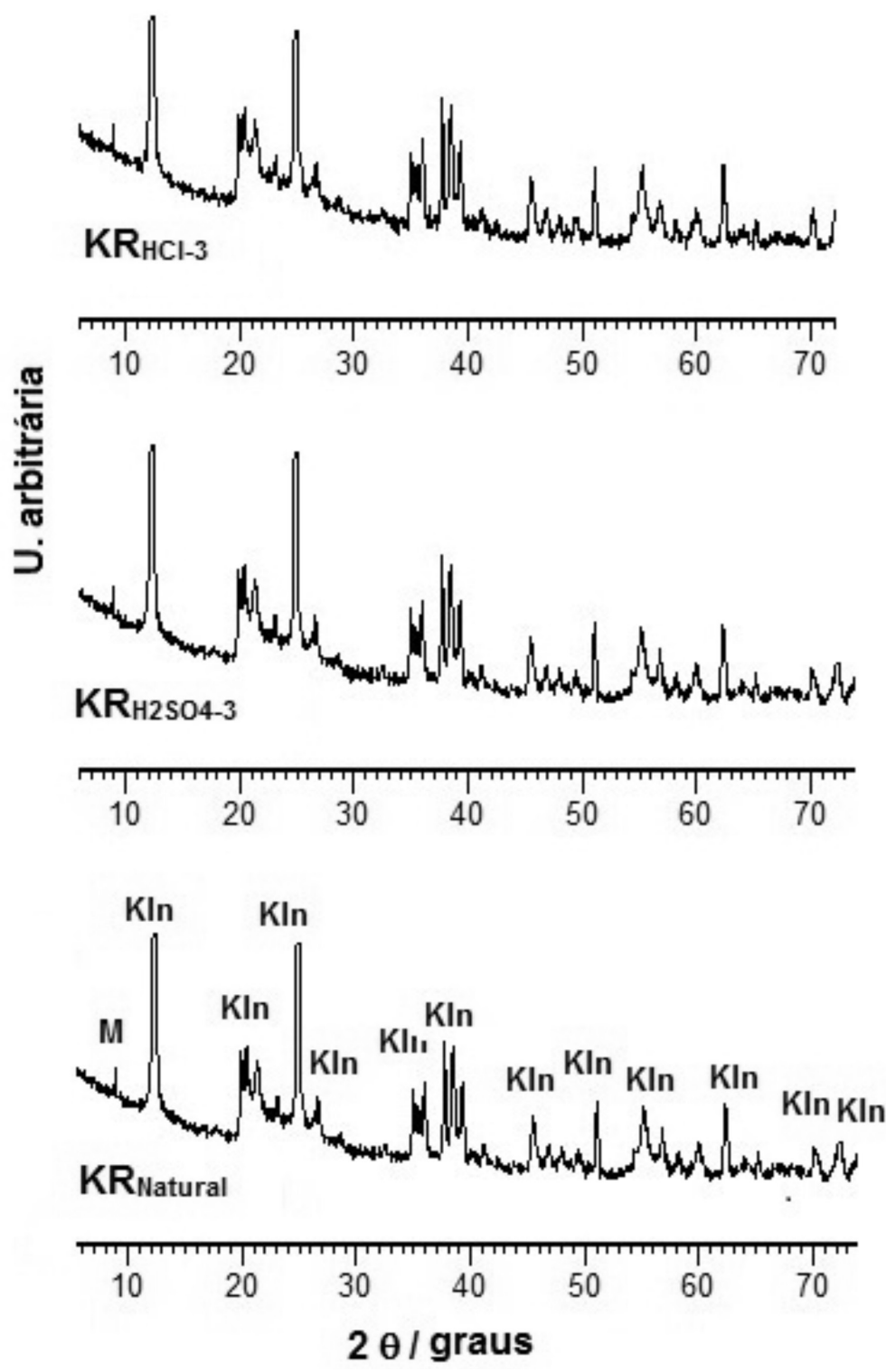

Figura 1S. Difratogramas de raios $\mathrm{X}$ do rejeito de caulim natural ( $\left.\mathrm{KR}_{\text {natural }}\right)$ e tratado com soluções de $\mathrm{H}_{2} \mathrm{SO}_{4}$ e $\mathrm{HCl} 3 \mathrm{~mol} \mathrm{~L}^{-1}$ ( $\mathrm{KR}_{\mathrm{H} 2 \mathrm{SO}-3}$ e $\mathrm{KR}_{\mathrm{HCl}-3}$, respectivamente). Kln indica a caulinita e $M$ a mica 

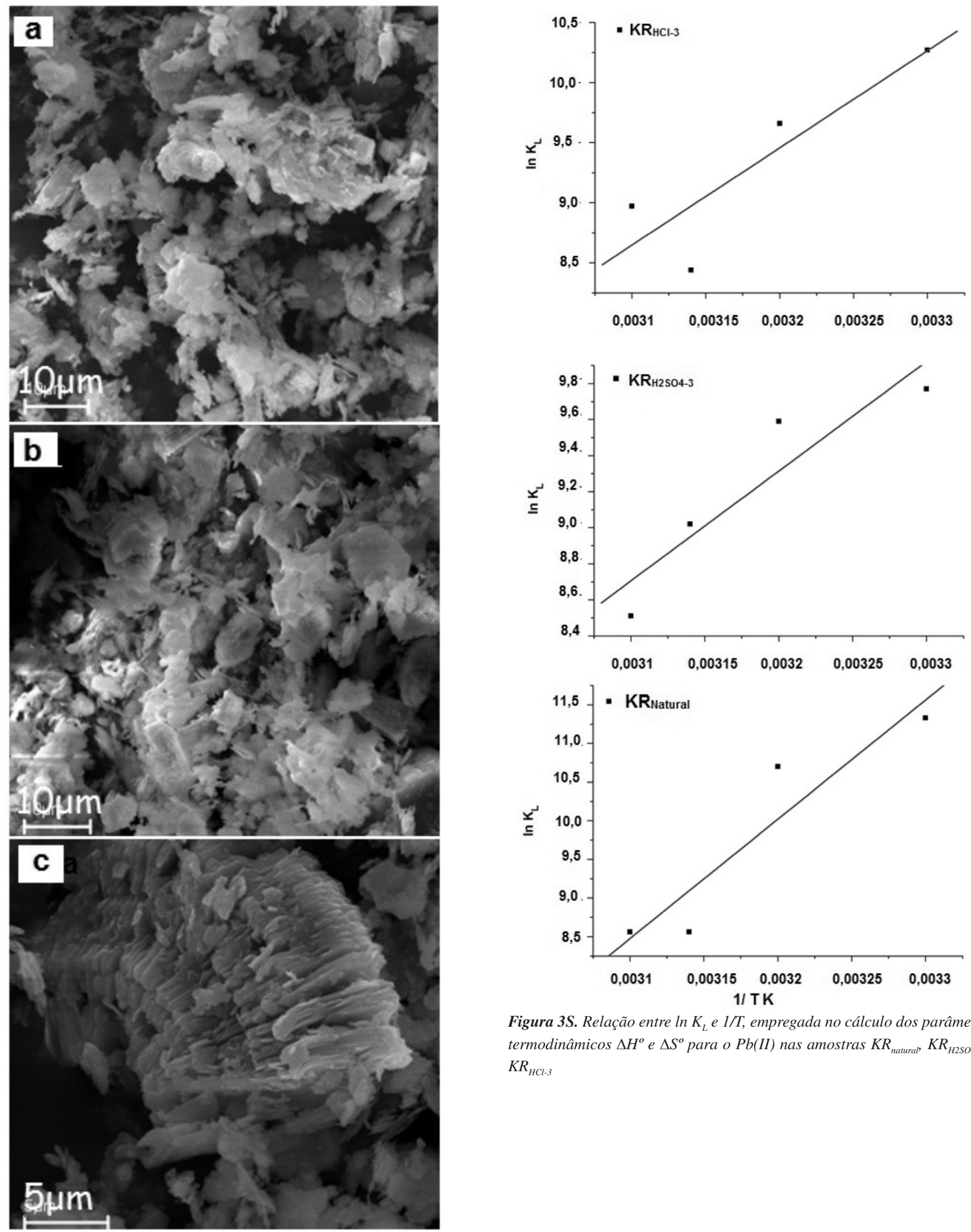

Figura 3S. Relação entre ln $K_{L}$ e 1/T, empregada no cálculo dos parâmetros termodinâmicos $\Delta H^{o}$ e $\Delta S^{o}$ para o $\mathrm{Pb}(\mathrm{II})$ nas amostras $K R_{\text {natura, }} K R_{H 2 \mathrm{SO4-3}} e$ $\mathrm{KR}_{\mathrm{HCl} \cdot 3}$

Figura $2 S$. Micrografias das amostras de rejeito de caulim: a) $K R_{H C l-3}$; b) $K R_{H 2 S O 4-3}$; c) $K R_{\text {natural }}$ 